\title{
Editorial
}

\section{Functional Devices for Clean Energy and Advanced Sensor Applications}

\author{
Ruomeng Yu, ${ }^{1}$ Caofeng Pan, ${ }^{2}$ Owen J. Guy, ${ }^{3}$ Lin Dong, ${ }^{4}$ and Matteo Tonezzer ${ }^{5}$ \\ ${ }^{1}$ School of Materials Science and Engineering, Georgia Institute of Technology, Atlanta, GA 30332-0245, USA \\ ${ }^{2}$ Beijing Institute of Nanoenergy and Nanosystems, Chinese Academy of Sciences, Beijing 100083, China \\ ${ }^{3}$ College of Engineering, Swansea University, Swansea SA2 8PP, UK \\ ${ }^{4}$ School of Materials Science and Engineering, Zhengzhou University, Zhengzhou 450001, China \\ ${ }^{5}$ IMEM-CNR, sede di Trento-FBK, Via alla Cascata 56/C, 38123 Povo, Italy \\ Correspondence should be addressed to Ruomeng Yu; ruomengyu@gatech.edu
}

Received 29 February 2016; Accepted 1 March 2016

Copyright (C) 2016 Ruomeng Yu et al. This is an open access article distributed under the Creative Commons Attribution License, which permits unrestricted use, distribution, and reproduction in any medium, provided the original work is properly cited.

The recent global increase of awareness about the environment pollution and the limited resources which are being rapidly consumed needs solutions. Networks of sensors are needed to monitor in real-time the quality of water, air, and in general the health of the ambient we are living in. Methods to clean wastewater and requalify industrial waste are required in order to recover the scars from decades of consumerism. Novel ecofriendly technologies will allow increased production of green and sustainable energy and its safe storage and transportation.

Nanoscience and nanotechnology are powerful instruments helping to face these challenges. A good control on nanometric size of materials permits reaching a wide range of new physicochemical properties and addressing problems which could not otherwise be solved. This approach can be utilized in several ways in different contests and applications, as can be seen in this special issue.

$X$. L. Jiang et al. studied theoretically a refractive index sensor based on a long period grating written in a specially designed photonic crystal fiber. They used hexagonal pattern with 5 rings of air holes around the central one. Using the finite element method and incorporating anisotropic perfectly matched layers as absorbing boundary conditions, they were able to evaluate the real and imaginary parts of the leaky mode. The shift of the resonant wavelength is sensitive only to the refractive index in the larger holes of the outer ring, which can be filled with a selected analyte.
H. Fu et al. developed a method to realize $\mathrm{TiO}_{2}$ (or any other metal oxide) hollow spheres by using hydrothermally synthesized colloidal carbon spheres as template. By adjusting the growth temperature and duration, the diameter of the spheres could be changed from 350 to $600 \mathrm{~nm}$, whilst their wall thickness and specific surface area can be regulated by adjusting the concentration of inorganic precursor. The monodispersed hollow spheres with hierarchically pore structure can be used as catalyst, battery, gas sensor, and functional material in several applications.

$\mathrm{X}$. Wang et al. investigated, both theoretically and experimentally, a new type of environment-friendly montmorillonite, a clay mineral used to adjust rheology and fluid loss of drilling fluid. They chose a new kind of green and steady ionic liquid as the intercalated agent (1-hexadecyl3-methylimidazolium chloride monohydrate or $\mathrm{C}_{12} \mathrm{mimCl}$ ) and varied its concentration from 10 to $5000 \mathrm{mg} / \mathrm{L}$. Simulations and experimental data were compared and, changing the amount of intercalated $\mathrm{C}_{12}$ minCl, functional parameters of the composite material such as viscosity and thixotropic loop area were improved for application as green clay composite for drilling fluid system.

X.-F. Ren et al. theoretically studied some novel environment-friendly dyes for dye-sensitized solar cells. The density functional theory was used to explore four dyes based on triphenylamine-containing indoline, changing some donor functional groups. The authors investigated the optimized 
geometry of each dye, the electronic coupling interaction between the dyes and $\mathrm{TiO}_{2}$ nanoparticles, and the HOMO and LUMO energy levels. They calculated the absorption spectra, the photoelectric conversion efficiency, and the lightharvesting efficiency of the four dyes, finding that one of them is expected to have an improved energy conversion efficiency compared to the commercial D149 dye.

T. L. Tran et al. developed an integrated three-electrode DNA sensor inside a microfluidic microchamber. Polypyrrole nanowires with diameters ranging from 80 to $110 \mathrm{~nm}$ were electrochemically synthesized in situ on a Pt working electrode inside the sealed microchamber. Then DNA probe sequences were immobilized on the PPy nanowires using the adsorption method, obtaining a specific DNA biosensor. The device can detect down to $20 \mathrm{pM}$ of complementary DNA with a response time of few seconds at room temperature.

T. T. L. Dang et al. synthesized polycrystalline $\mathrm{NiO}$ nanowires via a two-step method consisting in a hydrothermal growth followed by a thermal annealing. Dropping the nanowires between two interdigitated electrodes, they fabricated sensitive and quick gas sensors with a good selectivity towards hydrogen.

The paper of X. Zhao et al. is an exhaustive review of the recent literature about $\mathrm{ZnO}$ nanowire based sensors. The manuscript examines experimental data and working mechanism of ohmic-contacted and Schottky-contacted $\mathrm{ZnO}$ nanostructures based sensors. Their findings demonstrate that Schottky barrier mechanism greatly improves (several orders of magnitude) the response of the sensors. Furthermore, the use of piezotronic effect to modulate the Schottky barrier height can lead to extremely high sensitivities.

L. Mei et al. investigated experimentally and through molecular simulation the cation exchange interactions in montmorillonite clays. They focused on montmorillonite with $\mathrm{Na}^{+}$and $\mathrm{K}^{+}$in the interlayer spaces, testing the absorption and substitution of these cations with $\mathrm{Cd}^{2+}$. This phyllosilicate mineral can indeed be used for the removal and recovery of toxic cadmium from industrial wastewater. They found that $\mathrm{Na}^{+}$can be thoroughly exchanged by $\mathrm{Cd}^{2+}$, whilst $\mathrm{K}^{+}$can only be partially replaced.

The manuscripts in the issue focus on diverse topics but have a transversal common point: nanoscience and nanomaterials at the service of real applications for a cleaner world. We think that these papers will give the readers useful information and good hints for a further development in the field.

Ruomeng Yu Caofeng Pan

Owen J. Guy Lin Dong

Matteo Tonezzer 

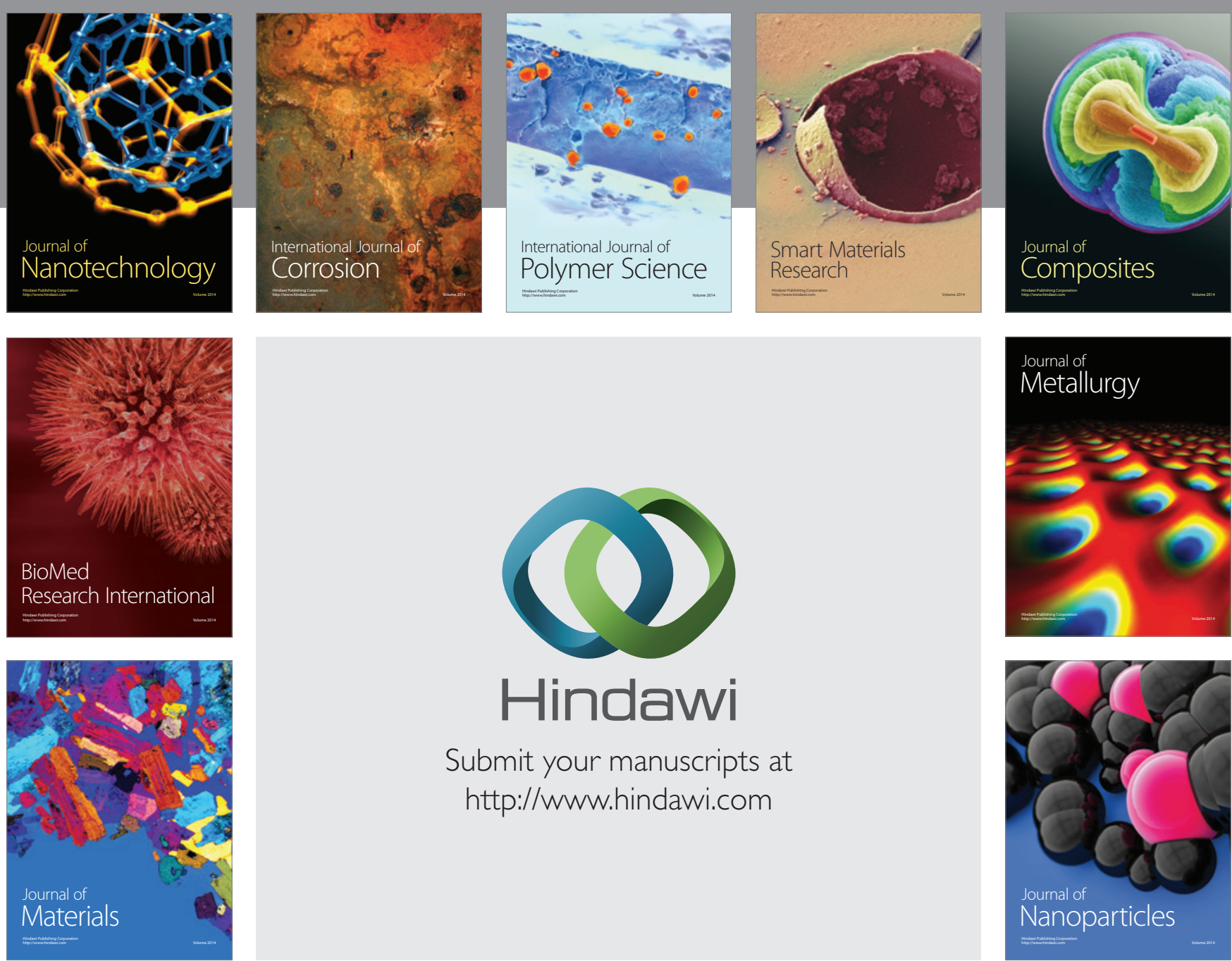

\section{Hindawi}

Submit your manuscripts at

http://www.hindawi.com

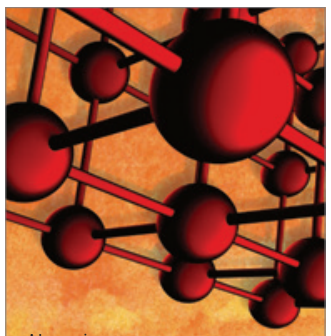

Materials Science and Engineering
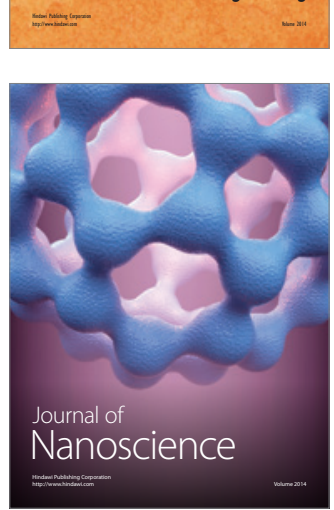
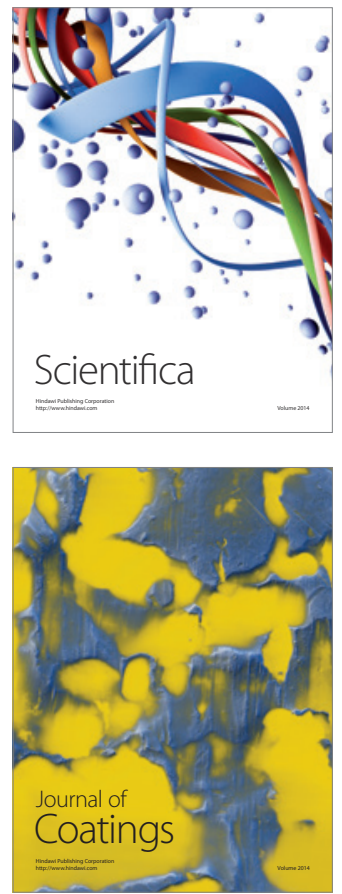
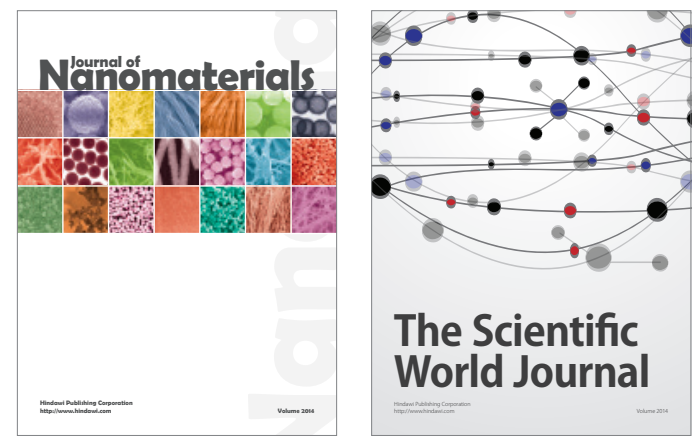

The Scientific World Journal
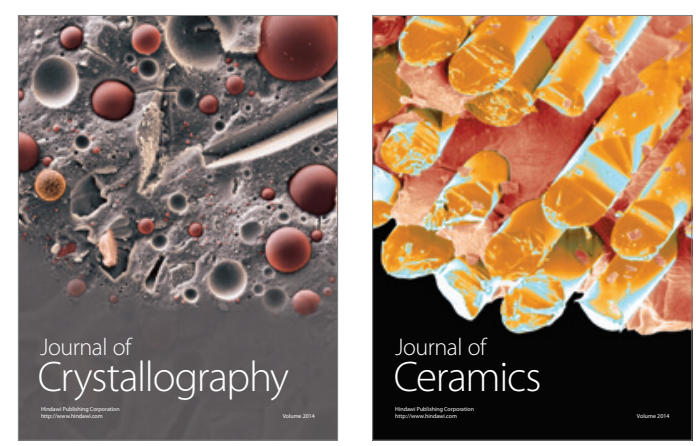
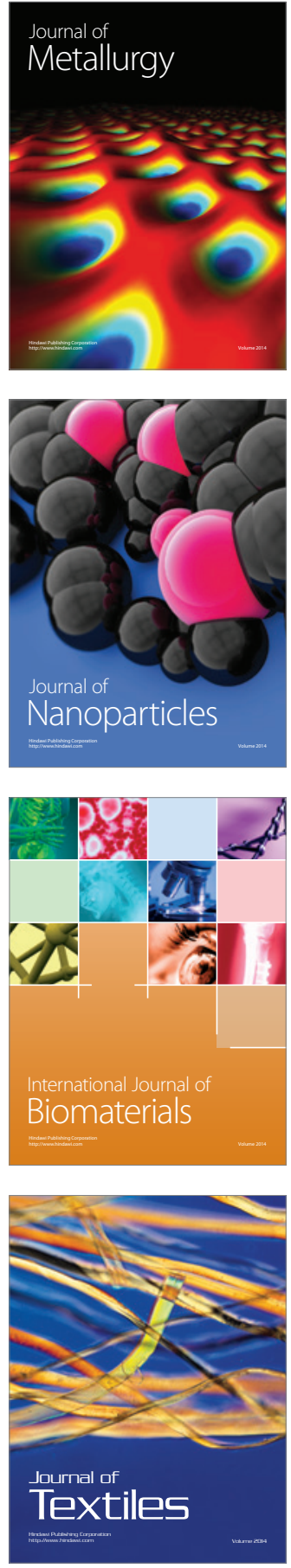